\title{
Detection of Bronchial Blood Flow from Earpiece Dye-dilution Curve
}

\author{
Ryo Katori, Kozui Miyazawa, Kinji Ishikawa \\ and Takashi Nakamura
}

\author{
The First Department of Internal Medicine (Prof. T. Nakamura), \\ Tohoku University School of Medicine, Sendai
}

\begin{abstract}
In an attempt to detect an increased bronchial blood flow in man from the earpiece dye-dilution curve, the reliability of three indexes was investigated by comparing them with the values of bronchial blood flow measured from the left atrial dye-dilution curve following dye injection into the aortic root. The first index is obtained from the $C_{p}$ method, in which the ratio of the peak concentration of early appearing dye-curve due to bronchial circulation to that of the dye-curve of the first circulation. The second is time index for appearance time of the early dye-curve, and the third is $C_{L} / C$, as a representation of elevation of the least dye concentration. $C_{p}$ method showed a good agreement with the bronchial blood flow irrespective of dye injection sites. The last two indexes were both correlated to the bronchial blood flow at a borderline level of significance. It was noteworthy that there was a sharp separation in the second index without any overlapping between cases with and without increased bronchial blood flow. These suggest usefulness of these indexes for clinical assessment of augmented bronchial circulation.
\end{abstract}

It has been elucidated that the bronchial artery markedly develops and makes a communication with the pulmonary circulation in various cardiopulmonary diseases. ${ }^{1-6}$ Since the bronchial artery originates from the thoracic aorta and most of its blood returns to the left atrium via bronchial-pulmonary anastomoses without passing the right side of the heart, the condition is equivalent to left-toright shunted blood flow in congenital heart disease. In fact, an early appearance of dye due to bronchial collateral circulation is demonstrated in the left atrium after dye injection into the aortic root. ${ }^{7,8}$ Therefore, it is likely that an increased bronchial blood flow gives a distortion to the peripheral dye-dilution curve as well as the left atrial dye-curve. ${ }^{9}$

The purpose of the present paper is to investigate the distortion of downslope of the earpiece dye-dilution curve for detecting an increase in bronchial blood flow in pulmonary disease and to compare the result with the values of bronchial blood flow measured by our previous method. ${ }^{8}$

Received for publication, September 30, 1969.

This investigation was supported by Research Grant HE 08018 from National Institutes of Health, U.S. Public Health Service. 


\section{Materials and Methods}

The study was performed in 20 adult patients with chronic pulmonary diseases, 10 of which had increased bronchial blood flow ranging from 3 to 12 per cent of the pulmonary blood flow when it was measured by the previously reported method (direct method), which will be shortly described later, and the remaining 10 did not show any increase in bronchial blood flow.

Earpiece dye-dilution curves were recorded following injection of $30 \mathrm{mg}$ of Coomassie blue into the femoral vein, main pulmonary artery, left atrium or aortic root, by using an Erma Dye-Densitograph ${ }^{10}$ connected to Sanborn Twin-Viso Cardiette.

In order to detect an increase in bronchial blood flow the $C_{p}$ method was used in the first place, which was reported previously from our laboratory ${ }^{9}$ for quantitating left-to-right shunt ratio from a single earpiece dye-curve in congenital heart disease. The principle is to estimate the concentration area of an abnormal hump due to shunted blood and to compare it with the area under the dye-curve of the first circulation. In practice, the ratio of bronchial blood flow to pulmonary artery blood flow was calculated by the equation $C_{p}$ '/ $\left(C_{p}-C_{p}^{\prime}\right)$, where $C_{p}^{\prime}$ is the peak concentration of the shunted dye-curve and $C_{p}$ is that of the dye-curve of the first circulation.

Secondly, the other two indexes were calculated from the earpiece dye-curve following femoral vein injection. One is $\left(t_{p}{ }^{\prime}-t_{a}{ }^{\prime}\right) /\left(t_{p}{ }^{\prime}-t_{p}\right)$ and the other is $C_{L} / C_{r}$ as shown schematically in Fig. 1 , where $t_{p}$ is the peak concentration time of the dye-curve of the first circulation, $t_{p}$ ' is that of the recirculation dye-curve, $t_{a}{ }^{\prime}$ is the appearance time of the recirculation dye-

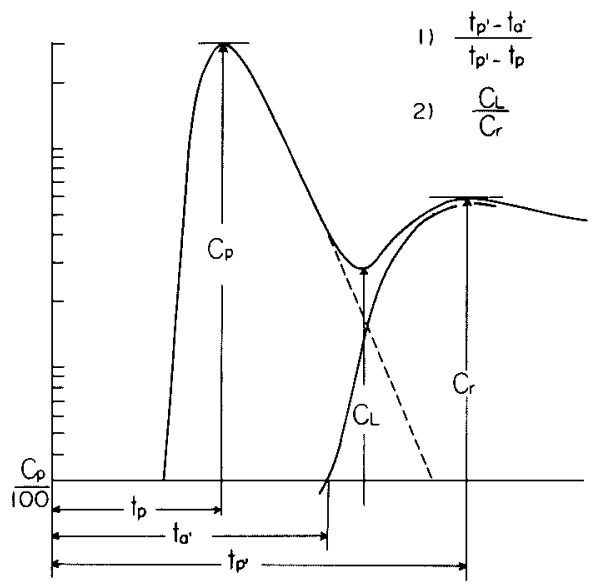

Fig. 1. Schematic representation of indexes in the dye-dilution curve for detecting increased bronchial circulation. The abscissa shows time in the linear scale and the ordinate dye concentration in the logarthmic scale. $C_{p}$ is peak concentration of the dye-curve of the first circulation, $C_{L}$ least dye concentration, $C_{r}$ peak concentration of the recirculation curve, $t_{p}$ peak concentration time of the dye-curve of the first circulation, $t_{p}$ ' peak time of the recirculation curve, and $t_{a}$ ' appearance time of the recirculation curve.

curve, $C_{L}$ is the least concentration and $C_{r}$ is peak concentration of recirculation dyecurve. When bronchial collateral circulation has developed, recirculated dye appears on the downslope earlier than the usual systemic recirculation owing to a short route of the collateral vessels, so that shortening of $t_{a}$ ' and increasing of $C_{L} / C_{r}$ may take place. For the calculation of the index $\left(t_{p}{ }^{\prime}-t_{a}^{\prime}\right) /\left(t_{p}{ }^{\prime}-t_{p}\right)$, dye-curve is replotted on the semilogarithmic paper so that recirculated dye is extrapolated by a straight line and $t_{a}$ ' is estimated at a concentration of one-hundredth of the peak concentration. When $t_{p}$ ' was ambiguous in 


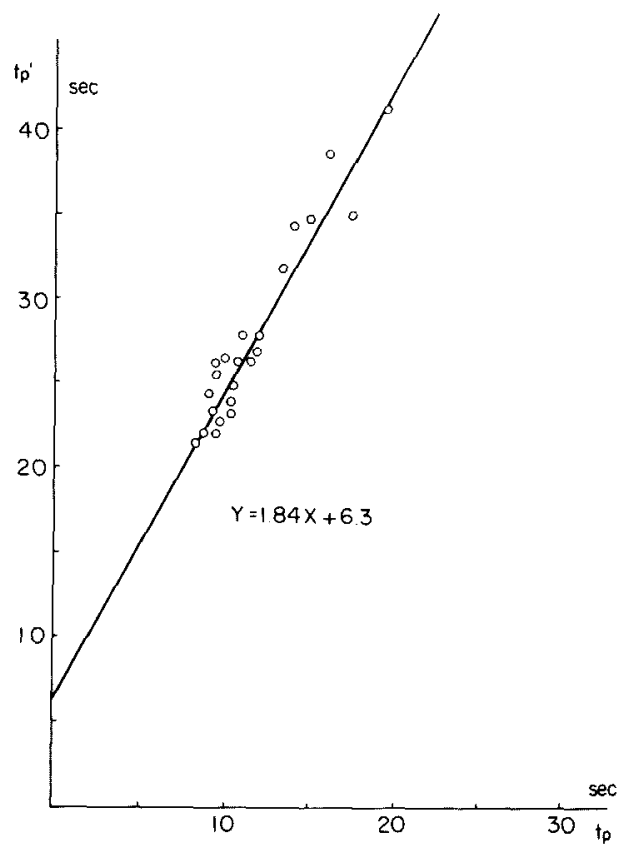

Fig. 2. Relation between peak concentration time of the first circulation dye-curve $\left(t_{p}\right)$ and peak time of the recirculatin curve $\left(t_{p}{ }^{\prime}\right)$ from earpiece dye-dilution curves after dye injection into the femoral vein in 23 adult patients without circulatory anomalies.

cases of increased bronchial blood flow owing to a great effect of early circulation dye on the downslope, $t_{p}$ ' was estimated from $t_{p}$ by using an equation, $t_{p}{ }^{\prime}=1.84 t_{p}+6.3$. The equation was derived from the earpiece dye-dilution curves following femoral vein injection in 23 adult patients without circulatory abnormalities as shown in Fig. 2.

The values obtained by the above-mentioned three methods were compared with the values of bronchial blood flow measured by the direct method. ${ }^{8}$ The direct method consists of simultaneous recording of two dye-dilution eurves from the left atrium and the ear following dye injection into the aortic root as described in the previous report. ${ }^{\mathrm{B}}$

\section{Results}

Fig. 3 demonstrates a distortion of the downslope of earpiece dye-curves in a case of severe pulmonary hypertention due to diffuse pulmonary thromboarteritis. The dye-curves display obviously an abnormal hump on their downslope, when dye is injected into the pulmonary artery and left atrium. The bronchial blood flow measured by the direct method was $19 \%$ of pulmonary artery flow. In the dye-curve shown in the upper panel, in which dye was injected into the femoral vein, slow decay of the downslope and absence of recirculation peak are obvious, although the abnormal hump is not visible. At autopsy, this case showed markedly developed bronchial arteries accompanied by numerous anastomoses with the pulmonary arteries. 


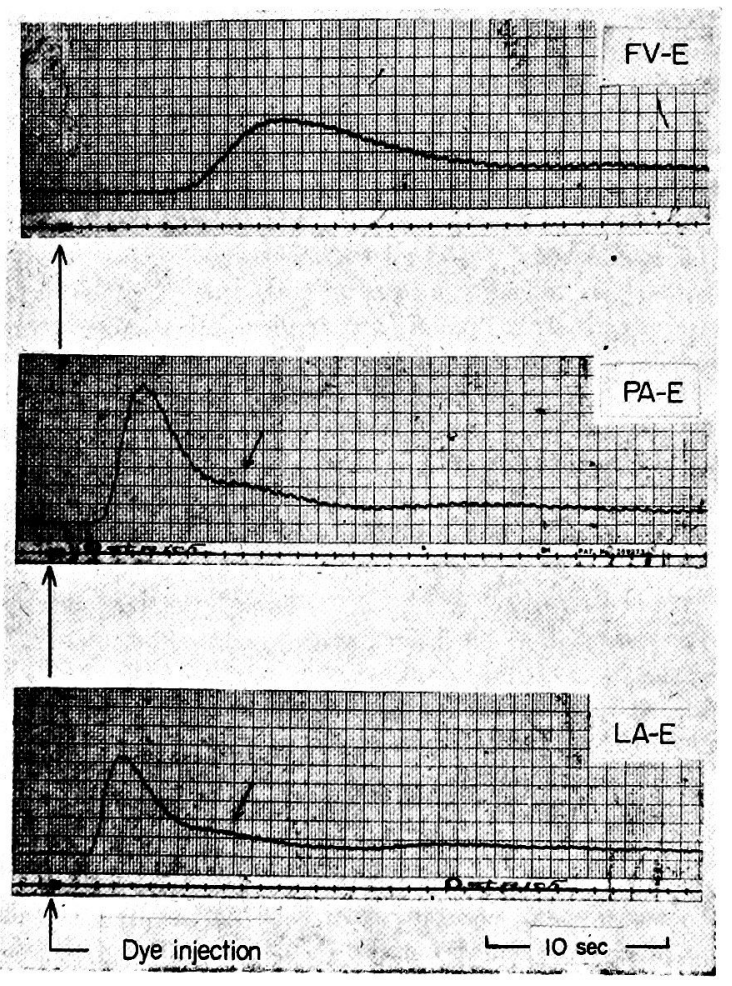

Fig. 3. Earpiece dye-dilution curves following dye injection into the femoral vein (upper panel), pulmonary trunk (middle panel), and left atrium (lower panel) in a 35-years-oldfemale patient with severe pulmonary hypertension due to diffuse thrombotic pulmonary arteritis. Bronchial blood flow amounted to $19 \%$ of pulmonary blood flow by the direct method. Arrows show abnormal hump on the downslope due to increased bronchial blood flow.

\section{Comparison of bronchial blood flow values by $C_{p}$ method and direct method}

The comparison between both methods was made in 4 dye injection sites as shown in Fig. 4. Significant positive correlations were obtained in all groups; the coefficient was 0.785 for femoral vein injection, 0.829 for pulmonary trunk, 0.781 for left atrium and 0.846 for aortic root. The correlation was significant at a level of $0.1 \%$ in all the groups.

\section{Comparison for two indexes and bronchial blood flow by direct method}

When two indexes were calculated from the earpiece dye-curve following dye injection into the femoral vein, correlations of a border-line significance with bronchial blood flow were revealed as shown in Fig. $5\left(r=0.585, p<0.05\right.$ for $\left(t_{p}\right.$ '$\left.t_{a}^{\prime}\right) /\left(t_{p}{ }^{\prime}-t_{p}\right), r=0.538, p<0.05$ for $\left.\mathrm{C}_{L} / \mathrm{C}_{r}\right)$. It is highly interesting that the cases with increased bronchial blood flow showed a sharp contrast to the cases without 


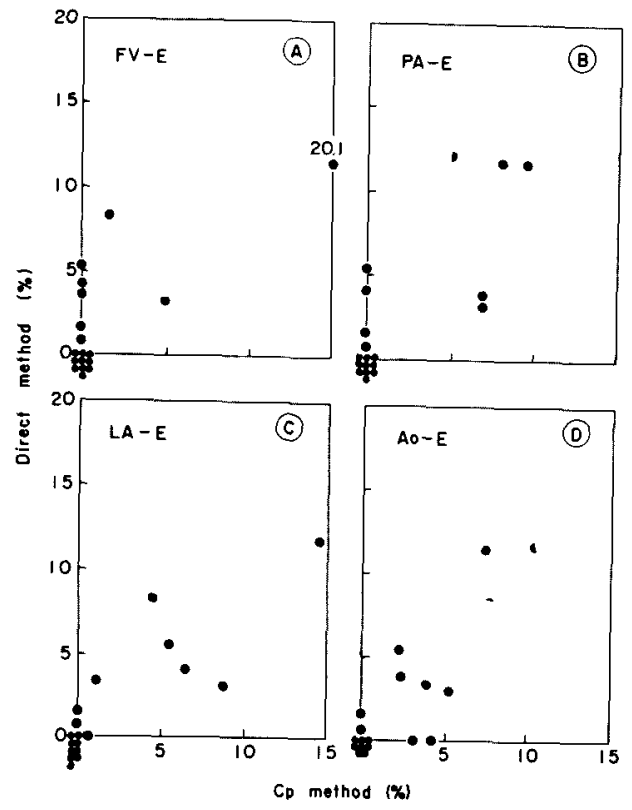

Fig. 4. Relation between the ratio of bronchial blood flow to pulmonary blood flow by the $C_{p}$ method (abscissa) and direct method (ordinate) in 20 cases with pulmonary disease. $A$ is that from dye injection into femoral vein, $B$ into pulmonary trunk, $C$ into left atrium, and $\mathrm{D}$ into aortic root.
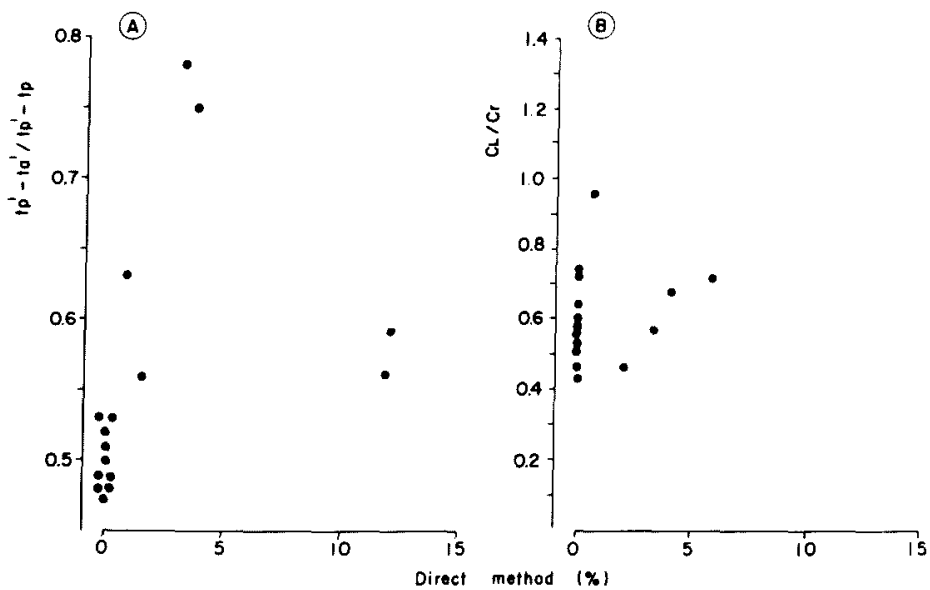

Fig. 5. Relation between bronchial blood flow by direct method and two indexes, $\left(t_{p}{ }^{\prime}-t_{a}{ }^{\prime}\right) /$ $\left(t_{p}^{\prime}-t_{p}\right)$ and $C_{L} / C_{r}$, calculated from the earpiece dye-curve following dye injection into the femoral vein. 
increased flow in the index $\left(t_{p}{ }^{\prime}-t_{a}{ }^{\prime}\right) /\left(t_{p}{ }^{\prime}-t_{p}\right)$; i.e., the index was more than 0.56 in all of the former, while less than 0.55 in all of the latter as seen in the left panel.

\section{Discussion}

Nakamura et al. ${ }^{8}$ measured bronchial blood flow in man by means of the dye-dilution method, in which the dye-dilution curve was recorded from the left atrium after dye injection into the aortic root. The method is based on an assumption that the circulation time through the collaterals must be shorter than that of the usual recirculation including systemic and pulmonary circulations, so that an early circulated dye through bronchial circulation can be detected prior to the recirculation of dye when the dye-curve was recorded in the left atrium. The validity of this assumption has been proved concerning circulation time in the previous report, ${ }^{8}$ i.e., appearance time of dye in the left atrium after aortic root injection was $3.1 \pm 0.8$ sec in cases with increased bronchial blood flow, while $7.6 \pm 1.5 \mathrm{sec}$ in cases without any increase in the flow. If the amount of bronchial blood flow is large enough to give an abnormal hump on the downslope of the dyecurve, calculation of the bronchial flow is possible in a manner similar to the estimation of left-to-right shunt from a single earpiece dye-curve. ${ }^{9}$ The abnormal hump and distortion of the downslope apparently indicate the possibility for quantitation of the flow as shown in Fig. 3.

Significant correlations were found between the values of bronchial blood flow measured by the $\mathrm{C}_{p}$ method in this study and the direct method irrespective of dye injection sites. This apparently indicates usefulness of the earpiece dye method for calculation of bronchial blood flow. However, several false positive and false negative cases were found with every injection site besides a slight difference in the result among the sites. Therefore, the $C_{p}$ method is not always adequate for quantitation of bronchial blood flow from the earpiece dye-curve in cases of a small bronchial blood flow.

The other two indexes are based on the detection of early appearance time of recirculated dye and rise of the least dye concentration of the downslope due to dye flowing through the bronchial circulation, i.e., $\left(t_{p}{ }^{\prime}-t_{a}{ }^{\prime}\right) /\left(t_{p}{ }^{\prime}-t_{p}\right)$ is for the former and $C_{L} / C_{r}$ is for the latter. The value of the former index was 0.5 on the average in cases without increased bronchial circulation, implying that recirculated dye appeared at the mid-point between peak times of dye-curves of the first circulation and recirculation. On the other hand, this index apparently rose to higher than 0.56 in all cases with increased bronchial blood flow and there was no overlapping on the value of this index between both groups. It is apparent that this index is useful for detecting the increase of bronchial blood flow. Since the detection is possible by dye injection into the peripheral vein and recording of the earpiece dye-curve, this index has an advantage in the clinical application. $C_{L} / C_{r}$ was increased in cases with a large bronchial blood flow, but there was a number of cases of overlapping between its value and bronchial blood flow, so that this index 
is not always suitable for detection of bronchial circulation, especially in cases of moderate increase in bronchial blood flow.

\section{SUMMARY}

An increase in bronchial blood flow was proved to cause a distortion on the downslope of the earpiece dye-curve. The distortion was quite similar to that in congenital heart disease with left-to-right shunt.

Measurement of bronchial blood flow was made by the $C_{p}$ method. The peak concentration was compared between the dye-curve of the first circulation and the shunted dye-curve using the earpiece dye-curve in 20 cases of pulmonary disease. Significant correlation was obtained between the values by this method and previously reported direct method irrespective of dye injection sites.

Two indexes were analyzed for detecting an increased bronchial blood flow from the earpiece dye-curve after dye injection into the peripheral vein. One was the detection of early appearance time of the shunted dye and the other was the ratio of least concentration to recirculation peak as indicating an elevation of the least concentration due to shunted dye. They correlated with the values of bronchial blood flow, although the correlation was statistically not of high significance level. The former index was considered apparently useful for clinical assessment of increased bronchial circulation, because of a sharp separation without any overlapping in the value of the index between cases with and without increased bronchial blood flow.

\section{References}

1) Wood, D.A. \& Miller, M. The role of the dual pulmonary circulation in various pathologic conditions of the lungs. J. thorac. Surg., 1937-1938, 7, 649-670.

2) Liebow, A.A., Hales, M.R. \& Lindskog, G.E. Enlargement of the bronchial arteries and their anastomoses with the pulmonary arteries in bronchiectasis. Amer. J. Path., 1949, 25, 211-230.

3) Marehand, P., Gilroy, J.C. \& Wilson, V.H. An anatomical study of the bronchial vascular system and its variations in disease. Thorax, 1950, 5, 207-221.

4) Cudkowicz, L. The blood supply of the lung in pulmonary tuberculosis. Thorax, $1952,7,270-276$.

5) Cudkowicz, L. \& Armstrong, J.B. The blood supply of malignant pulmonary neoplasms. Thorax, $1953,8,152-156$.

6) Viamonte, M., Parks, R.E. \& Smoak, W.M., III. Guided catheterization of the bronchial arteries. Radiology, 1965, 85, 205-223.

7) Nakamura, T., Katori, R., Miyazawa, K., Oda, J. \& Ishikawa, K. Measurement of bronchial blood flow in tetralogy of Fallot. Circulation, 1967, 35, 904-912.

8) Nakamura, T., Katori, R., Miyazawa, K., Ishikawa, K., Yamaki, M. \& Kobayashi, Y. Aortopulmonary collateral flow in patients with pulmonary disease. Amer. Rev. resp. Dis., 1968, 98, 464-473.

9) Nakamura, T., Katori, R., Watanabe, Te., Watanabe, Ta., Miyazawa, K., Murai, M., Oda, J. \& Ishikawa, K. Quantitation of left-to-right shunt from a single earpiece dye-dilution curve. J. appl. Physiol., 1967, 22, 1156-1160.

10) Nakamura, T., Katori, R. \& Watanabe, T. The validity of a photoconductive-cell densitometer for recording dye dilution curves. Tohoku J. exp. Med., 1963, 80, 338345. 